\title{
Using GIS in the Management of Health Infrastructure within Kaduna Metropolis, Nigeria
}

\author{
Aliyu, Y. A. \\ Shebe, M. W. \\ Department of Geomatics, Faculty of Environmental Design, \\ Ahmadu Bello University, Zaria-Nigeria
}

\section{Doi:10.5901/mjss.2013.v4n12p125}

\begin{abstract}
One of the most important indices of defining general welfare and quality-of-life of people in the world is the physical and mental health of individuals. The major factors causing diseases are essentially spatial; that is, their distribution and concentration vary in different locations.GIS is a decision support system that helps the managers of public health make sound decisions at less cost. In this study, coordinates of health facilities were collected using handheld GPS. Attribute data regarding these facilities were acquired. The information obtained enabled the creation of a geospatial database in ArcGIS 9.3 software. The database was queried and the results analyzed. The results show that primary health care $(\mathrm{PHC})$ centres constituted just 4.61 percent of the entire health facilities within the metropolis, 10.53 percent were government owned while 89.47 percent were private owned. Buffer operation of $1.5 \mathrm{~km}$ for the various health facilities displayed areas that are neglected.
\end{abstract}

Keywords: GIS, Public Health, Infrastructure Mapping, Kaduna

\section{Introduction}

More than 150 years ago, public health experts realized the use of maps in analyzing the location of diseaserelated happenings. Many interrelated changes in the world such as urbanization, transportation and industrial development, population and life expectation growth, unsustainable agricultural development, etc. cause general and complex environmental problems that threaten the health of humankind seriously (Mesgari and Massomi, 2008). In 1840, Robert Cowan in Glasgow-England, used maps to show the relationship between crowd and incidence of yellow fever. He recognized that in regions where there is too much immigration, this disease was more epidemic. Also in 1843, he showed epidemically incidence of typhoid on a map which involved all of the infected houses (Burrough, 1986). Since then, GIS has been continuously used for the analyses of spatial health related data. During this period, the more GIS analytical capabilities were developed, the more advanced and comprehensive spatial models were developed by the collaboration of experts in both areas of GIS and epidemiology and health care.

The need for health care varies in space and so the organization of provision necessarily has a spatial component. Neither population totals nor population characteristics such as age, sex, income, occupation, fertility et cetera are uniform in space. In a like manner, the physical environment varies in characteristics from place to place and this invariably has implications for the pattern of demand for health care facilities. The spatial dimension is also important in utilization behavior since accessibility is a major determinant of the use of health care facilities (Onokerhoraye, 1997).

Data and information are vital at each stage of the emergency cycle in order to make informed decisions and develop targeted response programmes. Seemingly a simple task - the humanitarian environment poses great challenges to timely and effective information collection and management. 
Fragmented workflows and the process of translating information into timely decision-making is an ongoing and well-documented challenge. (King, 2005; Harvard Humanitarian Initiative, 2011). The health sector, like many others, struggles to make decision amongst a simultaneous deluge and paucity of health information. (Turoff, 2008). Studies have reported over $80 \%$ of health information are geographical in nature and timely health interventions depend upon where health facilities are located and the status of each identified facility (e.g., hospitals, primary health care centers, health posts) (William, 1987).

Nowadays, health and health care are considered as models and an important factor in the quality of life of individuals. In fact, the development of public health and diseases management plays a significant role in cultural, social and economical development of any society. The most important goals of each public health organization involve environment health, control of diseases, health education and prevention, medical and nursing actions for early diagnosis, control and management of diseases (Ghazban, 2003). In fact up-to-date information and adequate models are required to help decision makers decide regarding any parameters affecting public health.

Both human settlements and activities and factors causing human diseases spread geographically. Most of the pathogenic factors are universally epidemic and do not belong to a special region or area, while some of them may occur in specific regions. Such correlations make it necessary to study and compare the spatial distribution and pattern of both the diseases, the affected population and their assumed factors. Geographic information system (GIS) can be used to analyze and compare such patterns.

In 2000, a project in Kamataka was accomplished for dividing regions and specifying local domain of health area responsibility. Reason for performing this study, was referred to the disproportion between population of the region and location of health center. The final goals were to control and supervise health center operations in their responsibility region, to optimize use of available health resources and to cover clients' needs. Related data to service area of health center had been provided by PHC and SC institutes for all sections and regions. The result was a GIS with the ability of performing spatial analyses, such as: zoning regions, finding the best location for facilities (Wen Hsiang, 2000).

Keola et al. (2002) used GIS for examining effects of different factors on public health, showing disease distribution, performing specific analyses, visualization and provision of information on health care and also helping in different decision making. Data used in this study include: population data, data concerning infectious diseases and their occurrence locations. In this study, dependence of spreading disease on time was studied using statistical regression analyses. One of the advantages of this study is the simultaneous use of spatial and statistical analysis which provides powerful tool for decision making process. Among all examined diseases, pneumonia had a direct relation with time and highest dependence coefficient (94 \%) and its distribution in crowded areas was high.

Eastern Europe International Health organization started to estimate diseases as a result of water pollution by means of GIS to specify pollution resources and direction of occurring diseases. In this research, primary studies determined system requirements for managing and taking care of diseases and also factors that cause them. Then, some of the disease intensifying factors and data related to them were gathered. Finally, GIS was used as a managing system to store and recover data, display and recognize temporal and spatial association of diseases (World Health Organization, 2002).

Ismaila and Usul, (2011) carried out a similar study in Yola, Nigeria and concluded that there is still gross inadequacy both in terms of health facilities and physicians and that GIS is an inevitable tool with several functions that can help health care planners in decision making process. It further recommended the need for an urgent improvement of health care delivery system in Yola for the betterment of people's lives and adoption of GIS technology by health care planners in Adamawa state.

Chan et al. (2012) also carried out a study whereby health facilities in Libya were mapped out. Results obtained state that 683 facilities were identified. The majority of facilities collected were from five locations (Tripoli, Benghazi, Az Zawiyah, Misratah, Sabha). Approximately $90 \%$ of identified facilities are part of the public sector. $57 \%$ of these facilities are hospitals, $25 \%$ are clinics, $14 \%$ are medical centres, and $2 \%$ for 
laboratory.

\section{The Study Area}

The study area covers an area of about $25 \mathrm{~km}$ long and $8-10 \mathrm{~km}$ wide from Kawo in the north to the oil refinery in the south. Kaduna metropolis comprises of four local government areas. They are Kaduna north, Kaduna south and part of Igabi and Chikun local government areas. A recent estimate puts the population at 1.5 million, but this must be compared to the 2002 satellite imagery, which shows 'on the ground' urban development more in scale with an overall population of around 4-500,000 (DFID, 2003).

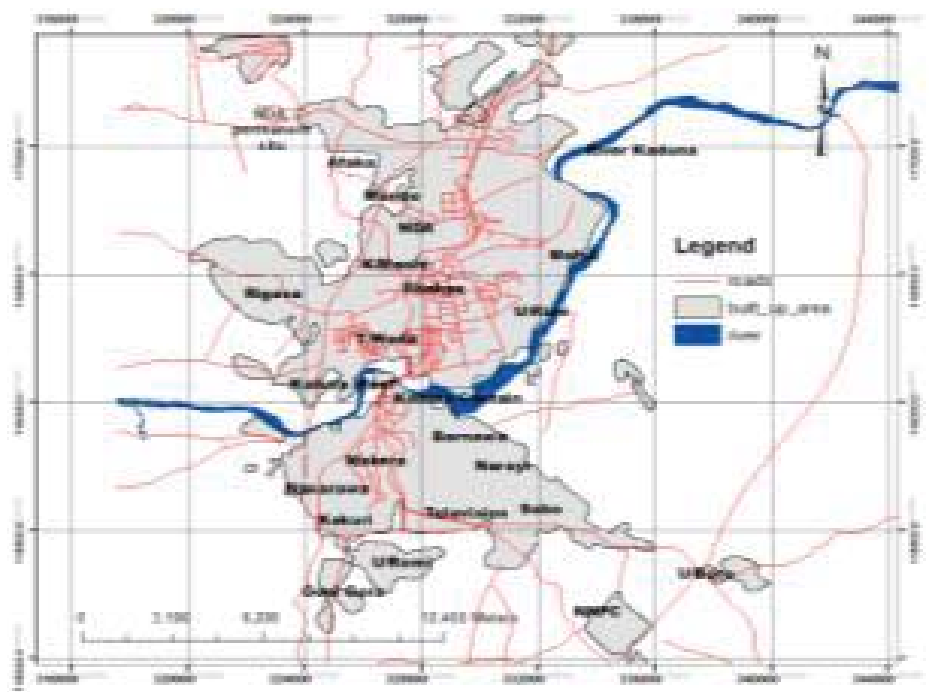

Figure 1: The Study Area

\section{Methodology}

A composite map of the study area was obtained from georeferencing and digitizing of land features from LandSat satellite image. A list indicating the names and addresses of health facilities was obtained from the state ministry of health. The locations of health facilities were plotted onto the base map using ArcGIS 9.3 software. A geodatabase was created with fields for name, address and number of bed for in-patients. The database was designed such that the positional data is well organized. It also provides for adequate linking, retrieval, updating of data.

The query was carried out using the query builder. The query builder tool is accessed by clicking on selection on the menu bar and pointing to 'Select By Attributes' and clicking. A dialog box appears. The query expression is built either by typing it in or clicking on the required fields, operators and values in the dialog box.

\section{Results and Discussion}

From the study, a total of 151 health facilities were identified. One hundred and thirty (130) were clinics, eight (8) were hospitals, seven (7) were primary health centres and six (6) were health laboratories. A display of the composition of health facilities within the Kaduna Metropolis is indicated in Figures $2-7$ below. 


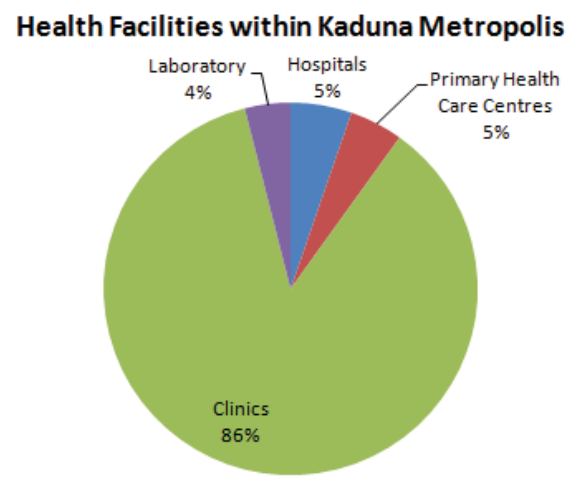

Figure 2: Pie Chart showing composition of health facilities within the Kaduna metropolis

The figures below display the results of the queries for private, government owned health facilities. Also query results for general hospitals, primary health care centers within the study area and as well as buffer analysis for these facilities to find out if they are adequate or not.

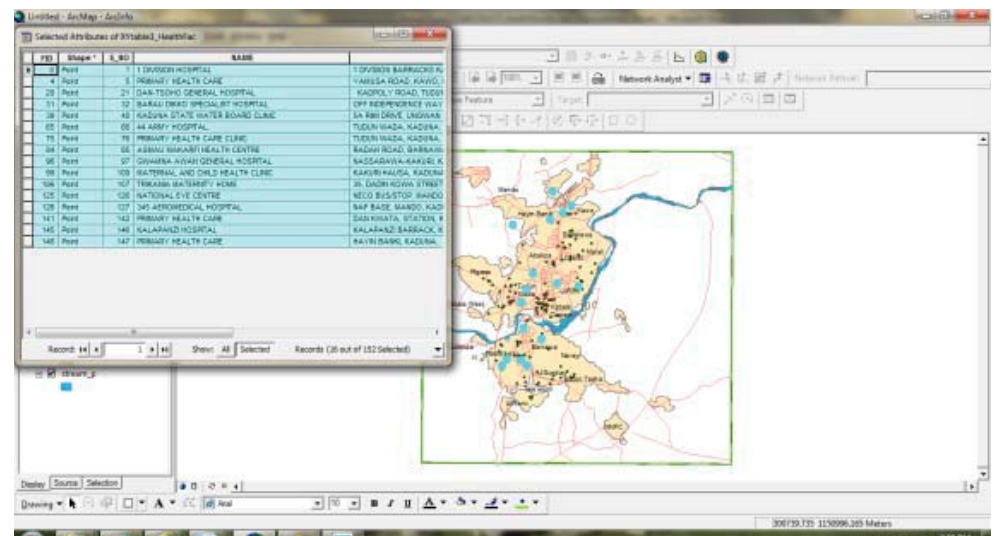

Figure 3: Results of query showing spatial distribution of government owned hospitals

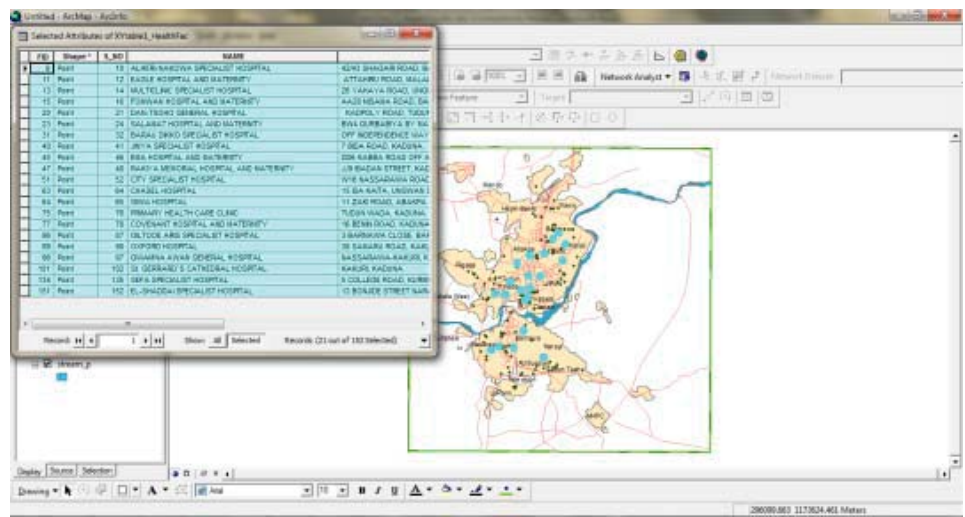

Figure 4: Results of query showing spatial distribution of health facilities with number of in-patient capacity of greater or equal to 20 


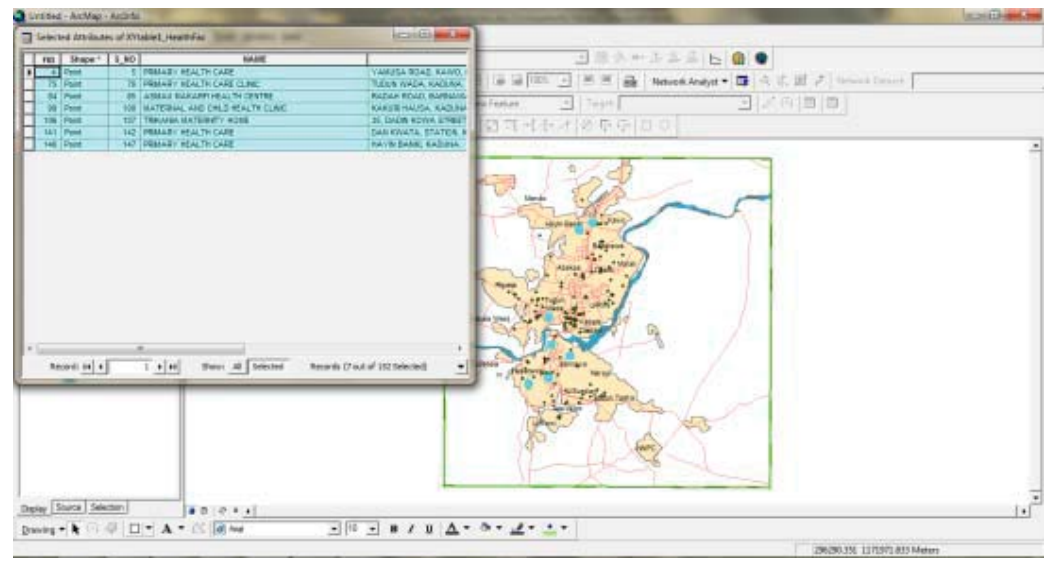

Figure 5: Results of query showing spatial distribution of PHC centers within the metropolis

From spatial display in Figure 5, areas such as Malali, Mando, Kudenda, Ungwan Romi, Ungwan Sunday, Sabon Tasha and Badarawa had no government recognized primary health centre (PHC). So it is recommended that these areas should appropriate government intervention especially regards to primary health care centres. All observed is that even among the recorded PHC, none of them had among its staff, a qualified medical doctor stationed in these facilities leaving patients at the behest of nurses/midwives/health officers.

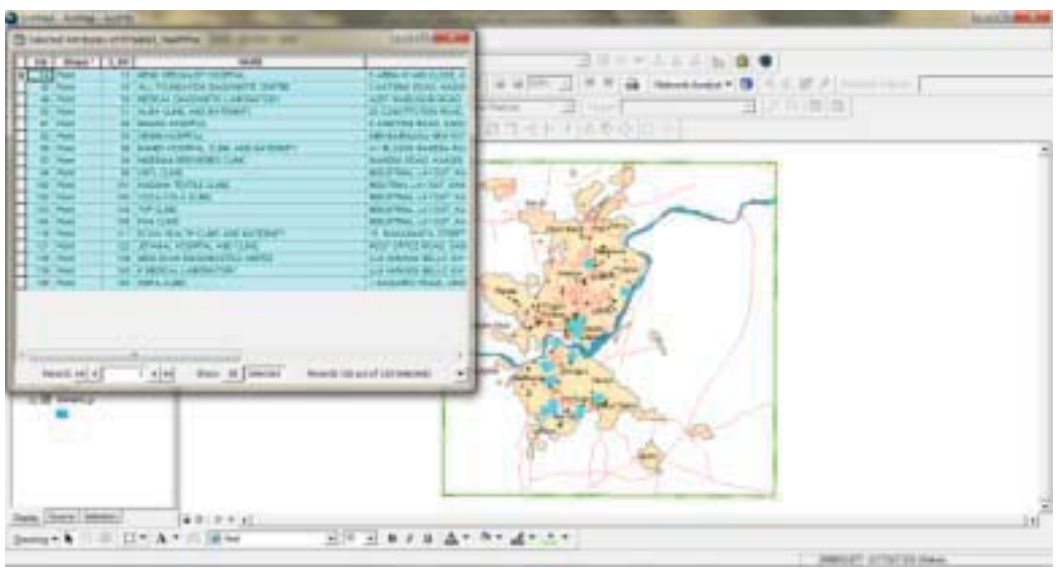

Figure 6: Results of query showing spatial distribution of private owned health facilities with no records of qualified resident doctors

Table 1: Results of Queries

\begin{tabular}{|l|c|c|}
\hline \multicolumn{1}{|c|}{ Theme of Query } & Number & Percentage \\
\hline Primary Health Facilities within the metropolis & 7 & $4.64 \%$ \\
\hline Government Owned health facilities & 16 & $10.6 \%$ \\
\hline Health facilities with (not less than) 5 qualified medical doctors & 14 & $9.27 \%$ \\
\hline Health facilities with less than 10 in-patient bed spaces & 90 & $59.6 \%$ \\
\hline
\end{tabular}


Using international criteria and local experience as guidelines (Rispel et al. 1995), the project team chose $2 \mathrm{~km}$ as the maximum distance a patient should be expected to walk to reach an urban health facility that provides a basic package of comprehensive primary care (Centre for Health Policy 1993). It was also assumed that this distance could be walked within half an hour. The distance of $2 \mathrm{~km}$ was translated into a radius of $1.5 \mathrm{~km}$ around a clinic in order to take account of the difference between actual walking distance and the straight line drawn on a map. Thus, a buffer operation was conducted with a radius of $1.5 \mathrm{~km}$ are carried out around existing health facilities in Kaduna metropolis, areas falling outside these buffers represent populations living too far from a health facility (Figure 5).

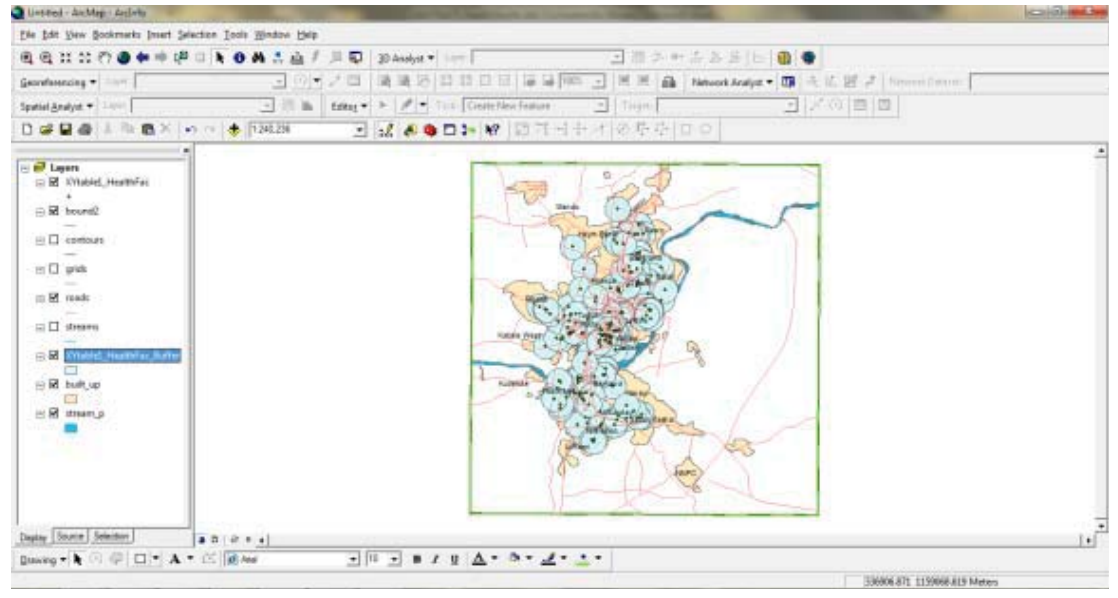

Figure 7: Buffer Operation for $1.5 \mathrm{~km}$ between health facility and built up area

From Figure 7, buffer operation of $1.5 \mathrm{~km}$ for the various health facilities, areas such as parts of Mando, Kudenda, Ungwan Romi and Mararaban Rido lacked availability of standard health facilities.

\section{Conclusion}

As a result of this study, it is concluded that the Kaduna state government need to establish more hospitals and primary health care centres in lacking areas. Furthermore, it should incorporate into its State Health Ministry, a GIS laboratory which will be useful in effective decision making.

\section{Recommendation}

- The Kaduna State Government should increase the number of primary healthcare centres within the metropolis as well as centres offering 24-hour services.

- More doctors and especially nurses should be appointed at the existing primary health centres since most of the patients are seen by nurses.

- Working conditions for staff should be improved by improving the health workers salaries and encouraging professional development, i.e. to train more nurses as primary health care practitioners and specialize in their field of interest. This would improve the quality of care.

\section{Limitation of the Study}

Due to time and financial constraints, it was impossible to include all the privately owned health facilities 
within Kaduna metropolis for the purpose of this study.

\section{References}

Burrough, P. (1986). Geographic Information Systems for Natural Resources Assessment. Oxford University Press, New York.

Centre for Health Policy. 1993. The Determination of Need Norms for Health Services. Part 2. A Summary of Norms in other countries. Document submitted to the Department of National Health and Population Development.

Chan, J. L., Colombo, R. and Musani, A. (2012). Mapping Libyan Health Facilities - A Collaboration between Crisis Mappers and the World Health Organization. Proceedings of the 9th International ISCRAM Conference Vancouver, Canada.

DFID, (2003). The Background of Kaduna in the Nigerian Federal Context. Mapping Urbanization for Urban and regional Governance. DFID Final Report - September 2003. Retrieved August, 6, 2013, from: http://home.wmin.ac.uk/MLprojects/Mapping/Report_for_Web/PDF_final/App_D_Kaduna_Back_MU.pdf

Harvard Humanitarian Initiative (2011) Disaster Relief 2.0 - The Future of Information Sharing in Humanitarian Emergencies, Washington, D.C. and Berkshire, UK. UN Foundation \& Vodafone Foundation Technology Partnership

Ghazban, F. (2003). Environmental-Biological Geology. Tehran University Press.

Ismaila, A. B. and Usul, N. (2013). A GIS-based Spatial Analysis of Health care Facilities in Yola, Nigeria. GEOProcessing 2013 : The Fifth International Conference on Advanced Geographic Information Systems, Applications, and Services.

Keola, S., Tokunaga, M., Nitin, K. T. and Wisa, W. (2002). Spatial Surveillance of Epidemiological Disease: A Case Study in Ayutthaya Province, Thailand. GIS Development Magazine.

King, D. (2005) Humanitarian Knowledge Management. Proceedings of the Second International ISCRAM Conference, Brussels, Belgium

Mesgari, M. S. and Masoomi, Z. (2008). GIS Applications in Public Health as a Decision Making Support System and its Limitation in Iran. World Applied Sciences Journal 3 (Supple 1): 73-77. ISSN 1818-4952

Onokerhoraye, A. G. (1997). Health and Family Planning Services in Nigeria: A Spatial perspective. University of Benin publishers. ISBN 9783282417

Rispel, L., Beattie, A., Xaba, M., Form, S., Cabral, J. and Marawa, N. (1995). A Description and Evaluation of Primary Health Care Services delivered by the Alexandra Health Centre and University Clinic. Johannesburg: Centre for Health Policy.

Turoff, M. and Hiltz, S. R. (2008). Assessing the Health Information Needs of the Emergency Preparedness and Management Community. Information Services \& Use, 28, 269-280

Wen Hsiang, W. (2000). Generalized Linear Models. Department of Statistics, Tunghai University, Tunghai.

William, R. E. (1987). Selling a Geographical Information System to Government Policy Makers. URISA, 3,150-6.

World Health Organization, (2002). Summit County Water Quality: Septic Systems and Potential Nitrate Pollution Analysis, URL:http://www.who.europe.int/ and http://ehasl.cvmbs.colostate.edu/projects/ 
